\title{
Die Informations- und Kommunikationstechnologien und die katholische Erwachsenenbildung
}

Ethisches Denken und christliche Verantwortung angesichts der Informationsgesellschaft

\author{
von Manfred Becker-Huberti, Düsseldorf
}

Eine Plastik in der Dortmunder Fußgängerzone ist mit folgendem Graffito besprüht: „So many people can determine the way you die, but only you can determine the way you live «. Wie wahr, kann man zu der gespritzten Kommunikation sagen, denn die Kassandra-Rufer, die Unheilspropheten haben Konjunktur. Nicht zuletzt aus der Bibel wissen wir, daß Zeiten des Umbruchs auch Zeiten der Unsicherheit sind; Zeiten der Neuorientierung sind auch immer Zeiten der Desorientierung. So ist es gegenwärtig in der Tat mindestens doppeldeutig, wie berauschend die kulturpessimistische Suada über jeden hinabstürzt, ihn einzuzwängen sucht in ein Lamento von resignativer Aufmüpfigkeit, geboren aus der Abwehr des Neuen und Erwartung des Chaos, oft sogar verständlich aus einer vorfindlichen Hilflosigkeit. Die Masse der Bevölkerung aber »ruhet sanft«; man fühlt sich an Lichtenberg erinnert: »Nichts kann zur Seelenruhe mehr beitragen, als wenn man gar keine Meinung hat $\alpha$. Dies aber ist - so behaupte ich - weder christlich, noch für die katholische Erwachsenenbildung Sinnmitte der Arbeit. Der Weg, der beschritten werden muß, weil man Fragen und Probleme nicht ohne Antwort lassen kann, also der verantwortbare Weg, ist der schmale Weg zwischen Panik und Ignoranz.

Diese Behauptung möchte ich mit einer kleinen Besinnung auf einige Elemente der christlichen Ethik belegen, um dann, nachdem kurz die allgemeine Aufgabenstellung der katholischen Erwachsenenbildung bedacht wurde, Ziele und Aufgaben eben dieser Erwachsenenbildung zu beschreiben, die sich aus unserer Fragestellung ergeben.

\section{Anfragen an die christliche Ethik ${ }^{1}$}

Seit Hiroshima ist es offenkundig: der Fortschritt hat mindestens zwei Seiten; wir wissen heute, daß der Fortschritt (oder Teilaspekte davon) unsere Welt unveränderlich schädigen kann. Tschernobyl ist hierfür der jüngste Beleg seitens der Basistechnologie Kernenergie; die Praktiken der Gentechniker, die gegenwärtig in die öffentliche Diskussion geraten sind, belegen dies für die dritte Basistechnologie, die Bio-Technologie; für die Informations- und Kommunikations(IuK)-Techniken, einen Teil der zweiten Basistechnologie, der Mikroelektronik, sind so augenfällig beweisbare Schädigungen (noch?) nicht nachweisbar. Es ist allerdings zu fragen, ob die leisen, stillen Veränderungen uns nicht jetzt schon gravierend ändern.

Aus einer in der Vergangenheit oft durch nichts zu ersetzenden Schicksalsergebenheit ist heute in einigen Fällen die durch Fortschritt bedingte Selbstbestimmung und Fremdbestimmung geworden, aus der heraus Menschen handeln. Aber wer darf wie und wonach handeln? Muß der Mensch - und wenn ja, vor wem? - sein Handeln begründen?

Hier besteht der eigentliche Konflikt: die Spannung zwischen dem technisch Machbaren und dem sittlich Verantwortbaren, dem Gekonnten und dem Gesollten. Dieser Konflikt ist nur durch ein neues Verständnis des Verhältnisses von Technik und Humanität zu lösen.

Dr. theol. Manfred Becker-Huberti ist katholischer Theologe und Publizist. Er leitet die katholische Bildschirmtext-Redaktion Düsseldorf, eine Einrichtung der Bistümer in Nordrhein-Westfalen. Der Autor gehört zur Redaktion von "Communicatio Socialis«. 
Wenn nicht mit ungebremster Zwangsläufigkeit das Gekonnte immer auch das Gesollte sein soll, wenn nicht der Erfolg oder die Akzeptanz einer Sache auch schon ihre Begründung darstellt, der ökonomische Gewinn zur legimatorischen Basis werden soll, dann muß definiert werden, was von dem, was wir können, auch zu verwirklichen ist. Angesichts einer nahezu grenzenlosen Steigerung der Machbarkeit und einer wachsenden Beherrschbarkeit der Natur erkennen wir, daß der Fortschritt sich nicht durch sich selbst legitimiert, daß Forschung nicht das Gute determiniert, Technik, so wie jedes andere Mittel auch, einer humanen Zielsetzung unterworfen werden muß, soll sie beherrschbar bleiben.

Im Verlauf der menschlichen Entwicklung wurde die Natur fortschreitend menschlichen Zwecksetzungen unterworfen; dennoch war sie bis in die Gegenwart etwas, was den Menschen umgreift und bestimmt. Noch heute ist der Mensch der Natur ausgeliefert und von ihr abhängig. Mit dieser Natur, in der der Mensch ja selbst auch Glied ist, lebte der Mensch über Jahrtausende in Symbiose.

Aus dieser Symbiose von Mensch und Natur ist während der Industrialisierung eine progressive Herrschaft des Menschen über die Natur geworden. Handelte der Mensch bis dahin dadurch, daß er die aus dem Umgang mit der Natur gewonnenen Erkenntnisse anwandte, so erarbeitet der Mensch heute sein Wissen durch Hypothesen. Die Nachahmung der Natur ist abgelöst durch die Einsicht in die Natur, die Entstehungsprozesse der Natur werden rekonstruiert, um die Eigenschaften des vorgegebenen Stoffes zu verstehen und zu gestalten. Aus bloß wissenschaftlichem Interesse wurde konstruktives Interesse, technisch-praktische Daseinsbewältigung. Die Natur wurde zum Objekt des Menschen.

Technik ist deshalb heute nicht bloß nachträgliche »Anwendung « der Naturwissenschaften, sondern ausgerichtet auf Welteroberung, Umgestaltung, Herstellung: Technik ist so die Fähigkeit, Vorgegebenes mit natürlichen oder künstlichen Mitteln auf einen vorgegebenen Zweck hin umzugestalten.

"Wir schreiten zurück, wenn wir nicht fortschreiten, weil man nicht stehenbleiben kann«, bemerkt Leibniz an den Rand der Lektüre des Buches "Wissenschaft der Theologie van Helmonts«. "Stillstand ist Rückschritt « popularisierte der Volksmund diese Erkenntnis, die, auf die Machtebene übertragen, lautet: »Wenn die Macht nicht vermehrt wird, muß man fürchten, sie zu verlieren«(vgl. Hobbes: Leviathan I, S. 11). Der Fortschritt ohne Regression prägt seit der Industrialisierung alle menschlichen Lebensbereiche; er vollzieht sich als Forschung, Entdeckung und Konstruktion, unterwirft die Natur immer mehr menschlicher Herrschaft und führt zum Verständnis einer wachsenden Befreiung des Menschen von der ihn »versklavenden Natur « und allen anderen Bedingungen. Die Aufhebung auch noch der letzten Naturgesetze ist danach nur noch eine Frage der Zeit. Fortschritt ist nicht mehr bloß erkennende Betrachtung der unverfügbaren Natur und Welt; menschliche Geschichte ist zu einer unaufhaltsamen Fortschrittsentwicklung geworden, die zielstrebig und unausweichlich zur Lösung aller menschlichen Probleme führt. Unausgesprochen oder aber als »Paradies auf Erden« prognostiziert, führt dieser Fortschrittsgedanke linear auf den Thron des göttlichen Geschichtssubjekts. Dem Fortschrittsgedanken wohnt nicht nur inne die gesamte Macht über die Natur zu erhalten, sondern selbst Herr der Natur zu sein. "Haben« und "Sein« zusammen ergeben erst die göttliche Dimension!

Innerhalb dieser Denkmuster sind schädliche Nebenwirkungen des Fortschritts akzeptabel, weil die »Errungenschaften « höher eingestuft werden als die Schäden. Eine Sensibilisierung für negative Fortschrittsfolgen ist schwer, weil eine am »Vorteil «orientierte Rationalität voranschreitet; die Anwendung der kommerziellen Prinzipien auf die Natur bele- 
gen die Überdüngung der Felder ebenso wie die Anwendung des Fließbandprinzips zur Produktionssteigerung in der Landwirtschaft.

Die ungelöste Problematik der Spannung zwischen Gekonntem und Gesolltem zeigt sich sowohl in angstvollen, teils hysterischen Reaktionen auf technische Großprojekte und Störfälle und die Propagierung von alternativen Technologien und alternativen Lebensstilenals auch in der bloß rationalen Reaktion der Technokraten, die nicht zu ahnen scheinen, daß sie Fragen beantworten, die keiner gestellt hat.

Inzwischen ersetzen die modernen Techniken immer mehr menschliche Funktionen, sie lassen ein »System zweiter Ordnung « entstehen, das Zwänge und Abhängigkeiten schafft. Natur und Mensch unterliegen einem Prozeß technischer Funktionalität und Rationalität. Was den einen aber noch als Natur- und Weltbeherrschung, als eine sich ständig ausbreitende Freiheiterscheint, als Autonomie des Menschen, trifft bei anderen auf herbe Kritik. Es wird gegenübergestellt:

Menschliche Autonomie: schwer durchschaubare Abhängigkeiten

Aufhebung materieller Not: Zwang zu immer neuen Bedürfnissen

Freiheit : »Entpersönlichung « und »Gleichschaltung «

Der aktuelle Diskussionsstand zeichnet sich dadurch aus, daß beide Standpunkte nicht harmonisierbar sind; kein Kompromiß besitzt genügend Tragfähigkeit für beide dualistische Sichtweisen.

Und noch etwas ist wichtig: Aus dem Spannungsverhältnis von technischer Machbarkeit und sittlich Verantwortbarem gibt es kein Entkommen: Die Freiheit der Forschung ist ein in der Verfassung garantiertes Grundrecht und wissenschaftliche Selbstbescheidung oder Selbstbeschränkung verlagern das Problem nur so wie die zum Teil propagierte »Verlangsamung der Forschung * auch. Dadurch, daß der Mensch sein Spannungsverhältnis zur Natur einseitig belastet hat, hat er seine Rolle als Teil der Natur aufgegeben, sich zum vermeintlichen Beherrscher der Natur befreit und die Natur dem Raubbau, der Ausbeutung unterworfen. Der Mensch hat zwei komplementäre Handlungsrichtungen ins Ungleichgewicht gebracht, die ihn in seiner Rolle als Teil der Natur bislang stabilisierten: auf der einen Seite das Gestalten und Konstruieren, ein schöpferisches Tun, das nicht sinnlose Vernichtung zum Ziel hat - und auf der anderen Seite das Bewahren, Behüten und Beschützen der Natur, das Schäden verhütet, Schutzräume bildet. Gestalten und Behüten sind aber die komplementären Verhaltensweisen eines Menschen, der Natur beherrscht, sich dennoch aber als einen Teil der Natur begreift. Das Zurückfinden zu dieser Position der Harmonie scheint zur Zeit der einzige Garant zur Überwindung des beschriebenen Grundkonflikts.

Gegenüber früheren Zeiten haben sich neue Bedingungen ergeben, über die wir uns klarwerden müssen, wenn wir diese Harmonie neu erstellen wollen:

* Heute ist die Natur als Ganzes Verantwortungsgegenstand; die Separation von Natur in Teilbereiche für die dann - unabhängig voneinander - Verantwortung übernommen wurde, ist überholt.

* Technik trägt heute vielfach das Potential der Unumkehrbarkeit in sich.

* Nutzung technischer Möglichkeiten obliegt nicht mehr einzelnen, sie sind kollektives Tun (zum Beispiel Autofahren, Umwelt et cetera). 
* Negative Auswirkungen der Technik betreffen nicht mehr nur einzelne, sondern alle und die zukünftige Existenz der Menschheit.

Wollte man das Gesagte in Form eines ethischen Imperativs verdeutlichen, so könnte man formulieren: Handle so, daß die Wirkungen deiner Handlungen nicht zerstörerisch sind für die künftigen Möglichkeiten menschlichen Lebens auf Erden.

Die Pfeiler für ein Ethos angesichts der Grundkonflikte der modernen Zivilisation lauten dann:

* Achtung der Würde des Menschen

* Anerkennung der Persönlichkeit des einzelnen

* Recht auf Leben

* Recht auf körperliche und seelische Unversehrtheit

* Selbstbestimmung des einzelnen Menschen bei gleichzeitiger Geltung des gleichen Rechtes für alle anderen Menschen

* Verpflichtung, den Menschen nicht als Mittel zum Zweck in Gebrauch zu nehmen

Eine andere Form der Betrachtung wäre die Gliederung in drei Bereiche:

* die gegenständlichen Bereiche:

Ethische Einsicht und Wissenschaft/Technik dürfen nicht als voneinander unabhängig betrachtet werden.

* die Rechtfertigung der Ziele:

Vernünftigkeit und Humanität der Ziele; Grundziele: soziale Sicherung, allgemeiner Wohlstand, Befreiung von den Zwängen niederdrückender Arbeit.

* Verantwortung von Folgen:

Menschliche Zukunft muß ethisch, nie nur technisch und bloß sozial bewältigt werden.

Dieser Rückblick auf die Problemsituation aus dem Blickwinkel der christlichen Ethik verlangt nach ihrem Ort. Daß wir fragen, was die katholische Erwachsenenbildung angesichts der IuK-Techniken tun soll, läßt uns nun vom allgemeinen zum besonderen kommen und fragen, ob, und wenn ja, wie die katholische Erwachsenenbildung hier eine Aufgabe hat.

\section{Neue - alte Ziele und Aufgaben der katholischen Erwachsenenbildung ${ }^{2}$}

(Bei dem Versuch, eine von breiter Übereinstimmung getragene Definition der Erwachsenenbildung zu finden, bin ich gescheitert, trotz einer überschwappenden Fülle an Litera-

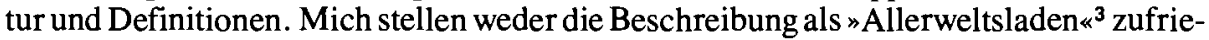
den, noch soziologische Begriffsakrobatik nach dem Muster: "Erwachsenenbildung in kirchlicher Verantwortung hat hinsichtlich der Primär-, Sekundär-und Resozialisation eine mehrfache Aufgabe, die je nach Situation einen affirmativen, komplementären, korrigierenden oder therapeutischen Charakter annehmen kann “ $^{4}$. Ich habe mir deshalb, ausgehend von der Theologie und kirchlichen Positionen in Verbindung mit langjähriger Mitverantwortung in der kirchlichen Weiterbildung eine Beschreibung gebildet, die pragmatisch in die Themenstellung paßt, ohne sich jedoch einer theologischen und pädagogischen Vertiefung zu entziehen.)

Katholische Erwachsenenbildung ist die organisierte Hilfe bei der Lebensgestaltung aus christlichem Glauben. Orientiert an Modellen des christlichen Menschenbildes, die an 
Zeit- und Umweltbedingungen angepaßt werden müssen, soll die Erwachsenenbildung den Menschen im Denken, Fühlen und Handeln zu vertiefter(m)

Ich-Findung (Identität)

Du-Fähigkeit (Partnerschaftlichkeit) und

Wir-Handeln (Sozialverhalten)

verhelfen. Seitens der katholischen Erwachsenenbildung meint Hilfe "Hilfe zur Selbsthilfe«. Seine Arbeit kann der Erwachsenenbildner näherhin beschreiben als:

* Dialog mit Menschen und Welt

* Teilnahme am kirchlichen Verkündigungsauftrag

* Wegweisung und Weggemeinschaft

* Auseinandersetzung mit konkreten Menschen und aktuellen Problemen

* Mithilfe bei der Verwirklichung eines erfüllten christlichen Daseins

Eben diese Form von Erwachsenenbildungsarbeit ist nicht zu leisten ohne

* die Sensibilisierung der Erwachsenenbildner für Tendenzen und Strömungen in der Gesellschaft, die Fragen aufwerfen und Antworten herausfordern

* die Hörbereitschaft und Dialogfähigkeit, verbunden mit dem - nie bloß professionellen - Willen, sich ganz einzubringen und Zeuge Christi zu sein. Katalysatorfunktionen der Pädagogen, bloße Wissensvermittlung, Unverbindlichkeit sind die Gegenbeweise für die Gültigkeit der Zielsetzung!

* Optimismus, Begeisterungsfähigkeit, Liebe zum Menschen, gelebtes "Prinzip Hoffnung « und unbändige Lust, das Gute und Begabungen in Menschen zu entdecken und zu aktivieren, sind Voraussetzung.

Mir scheint, daß nur so entscheidungsfähige Menschen als msoziokulturelle Persönlichkeiten« zur Reife zu bringen sind. Menschen, deren Denken, Fühlen und Handeln an der aktualisierten Botschaft Christi ausgerichtet ist, die das Miteinander-Tun in der Weiterbildung als ein Miteinander-Sein erleben und erfahren, das dieses Miteinander-(Er)-Tragen ermöglicht, so daß christliches Leben zur peregrinatio religiosa, zur Reise auf das letzte Ziel wird.

Wenn sich katholische Erwachsenenbildung so oder so ähnlich versteht, wie hier unterstellt wurde, dann ist es keine Frage mehr, ob sie sich den Anfragen der neuen Technologie stellt: es ist ihre selbstverständliche Aufgabe und Pflicht, will sie ihre Zielsetzung, Menschen aus christlichem Wertdenken zu begleiten, nicht verraten.

Weder das Unbehagen an neuartigen Sachfragen, noch die Unterbewertung der fachfremden und verwirrenden Technik, noch das zur Zeit vorherrschende Desinteresse der Bevölkerung, mit beunruhigenden Problemen befaßt zu werden, noch irgendetwas anderes darf da abhalten. Nicht Ignoranz oder Panik, nicht Lockangebote oder Anpassungsstrategien an modische Vorgaben sind gefragt, sondern sinn- und wertorientierte Angebote in Form von Lebenshilfe und Erweiterung von Handlungskompetenz. 
Wie läßt sich dies nun genauer konkretisieren? Die IuK-Techniken sind

* Gegenstand der katholischen Erwachsenenbildung (= Reflexionsgegenstand) und zugleich

* Denkzeug und Werkzeug (= Hilfsmittel zur Problemlösung).

Beide Dimensionen bedürfen der Thematisierung, zumal die bei weitem überwiegende Zahl der Erwachsenen bislang kaum Zugang zu diesen Techniken gefunden hat. Die isolierte Beschäftigung mit nur einem der beiden genannten Punkte führt leicht zu Fehlverhalten. Es kann nicht darum gehen, die katholische Erwachsenenbildung umzufunktionieren als Einrichtung zur Anpassung an Schnittstellen der Maschinen; ebenso unmöglich ist die bloße Beschäftigung mit dem theoretischen Hintergrund, ohne konkret $\mathrm{zu}$ wissen, wie solche Geräte aussehen und funktionieren.

In die vorhandenen Tätigkeitsfelder läßt sich diese neue Thematik einordnen

* als soziales Wissen/Können

* als Kommunikationspädagogik (wobei sie nicht, wie gehabt, zu einer Präventionspädagogik nach Art der inzwischen hoffentlich verblichenen »Medienerziehung« werden darf)

* als integrativer Bestandteil aller anderen Sachbereiche.

Dies zu ermöglichen ist Verpflichtung der Träger der katholischen Erwachsenenbildung; dies auch schon zu können und zu wissen ist deshalb noch lange nicht für die Erwachsenenbildner die Folge. Die Ausbildungsinstitutionen greifen gegenwärtig die genannten Probleme eher mißmutig und schwerfällig auf. Die Fortbildung der Erwachsenenbildner spart unseren Themenbereich weitgehend aus. Wenn ich es richtig einschätze, dann gibt es gerade unter den Erwachsenenbildnern eine Gruppe, die ihre Intellektualität dadurch nachzuweisen sucht, daß sie solche Themen ignoriert. Mit großer Dringlichkeit ist es angebracht, die Probleme der IuK-Techniken zum Gegenstand der Fortbildung zu machen. Für die Erwachsenenbildner selbst sind zu erreichen:

* Erwerb von Sachkompetenz unter Berücksichtigung unterschiedlicher Aspekte, zum Beispiel Technik, Soziales, Wirtschaft, Kultur und andere

* Erweiterung der fachdidaktischen Handlungskompetenz und Aufbereitung neuer Ziele und Inhalte.

Bei der Einrichtung von hausübergreifenden Fortbildungsmöglichkeiten wird man entscheiden müssen, in welchen Strukturen die Fortbildung angeboten werden soll, als

* Multiplikatoren- (= Moderatoren) Modell

* auf freiwilliger Basis für bestimmte Fachrichtungen

* auf freiwilliger Basis für alle Fachrichtungen.

Daneben ist die hausinterne Aufnahme der Thematik in die sicher allerorten stattfindende Supervision zu empfehlen.

Was sollen nun die Teilnehmer an Fortbildungsveranstaltungen lernen, wie läßt sich der Stoff auf sie hin konkretisieren? 
Ich stelle die nachfolgenden Lernziele zur Diskussion:

* Vermittlung und Förderung eines christlichen Menschenbildes, das unter den Bedingungen der Informationsgesellschaft Gültigkeit besitzt und Menschen zu eigenverantwortlichem Denken, Fühlen und Handeln freisetzt.

* Das Kennen- und Durchschauenlernen medialer Systeme und Zusammenhänge, das Umgehenlernen mit dieser Technik und das Erlernen der sozialen und kreativen Beherrschung der IuK-Techniken.

* Menschen müssen zwischen menschlicher und technischer Kommunikation nicht nur unterscheiden lernen, sie müssen auch die jeweiligen Vor- und Nachteile, die Eigengesetzlichkeiten und Folgen begreifen lernen (= Gestaltungswissen).

* Priorität muß der Entfaltung der typisch menschlichen Kommunikation zugewiesen werden (kommunikationspädagogischer Ansatz).

Das Gesagte und Angedeutete soll in einem Schaubild plastisch werden. Alle inhaltlichen Beschreibungen beanspruchen nicht den Status von ex cathedra-Entscheidungen, sondern sind zur Diskussion gestellt, damit sie ergänzt, ersetzt und verbessert werden können.

\section{Schlu $\beta$}

Ein Blick in die Geschichte, genauer noch in die Religionsgeschichte, zeigt, daß die sinnspendenden Institutionen, die in ihrer Zeit keine tragfähigen Antworten mehr geben konnten, mit ihrer Unfähigkeit ihr eigenes Todesurteil geschrieben hatten. Unsere Glaubwürdigkeit als Christen gründet sich in unserer Fähigkeit, aus unserer Sicht der Dinge überzeugende Lösungen anzubieten, also persönlich bezeugte Lösungsmodelle. Auch wir Christen haben nicht alle Antworten auf alle Fragen, jedoch dic Pflicht zur Solidarität auch dort, wo wir keine endgültigen Antworten haben, Weggefährte, Mitsucher, Betroffener zu sein. Wenn wir uns aus unserem Selbstverständnis heraus etwas nicht leisten können, dann ist das die Negation der Realität und ihrer Fragen und Probleme. Weder der kulturelle Hochmut des Übersehens der Aufgabenstellung, noch die hoffnungslose Resignation, die Flucht in den Kulturpessimismus sind hier Auswege. Nicht nur der Weg zum Heil führt durch ein schmales Tor; auch der Weg, den wir beschreiten müssen, ist ein schmaler Pfad. Der Weg zwischen Panik und Ignoranz ist der christliche verantwortbare Weg in die Informationsgesellschaft. Handeln wir!

\section{Anmerkungen}

Bei dem Artikel handelt es sich um das überarbeitete Manuskript eines Vortrages anläßlich der Jahrestagung der Bundesarbeitsgemeinschaft für Familienbildungsstätten, 13. - 15.5.1986, in Cloppenburg.

1 Die in diesem Abschnitt enthaltenen Gedanken basieren auf einem Vortrag des Mainzer Bischofs Karl Lehmann: Das technisch Machbare und das ethisch Verantwortbare. Überlegungen zu einem Grundkonflikt der modernen Zivilisation. Vortrag im Mainzer Dom innerhalb der Reihe „Für eine menschliche Zukunft«am 15. April 1986.

Vergleiche zum Thema auch die nachfolgend genannte (ausgewählte) Literatur:

Auer, A : Verantwortete Vermittlung, Bausteine einer medialen Ethik. In: Ethik und Kommunikation. Telekommunikation - ein Fortschritt für den Menschen? hrsg. v. Zentralstelle Medien der Deutschen Bischofskonferenz u.a., Stuttgart 1979, S. 61-80.

ders.: Ist Unterhal tung vertane Zeit? Überlegungen zur Unterhaltung in den Massenmedien aus der Sicht einer theologischen Ethik. In: Stimme der Zeit 190 (1980), S. 735-749.

ders.: Verantwortete Vermittlung. Neue Überlegungen zu einer medialen Ethik. In: Stimme der Zeit 199 (1981), S. $147-160$. 
Beaugrand, G.: Neue Medien und Familie. Familienpolitische, gesellschaftliche und pädagogische Aspekte der künftigen Telekommunikation. ( = Schriftenreihe des Familienbundes der Deutschen Katholiken, Landesverband NRW, Heft 3). Münster 1981.

Becker-Huberti, M.: Neue Medien - Neue Menschen? In: Communicatio Socialis 1/1986, S. 17-32.

Betz, G.: Die neuen Medien und die katholische Kirche. Positionen - Hintergründe - Anfragen. In: Religionspädagog. Beiträge 15 (1985), S. 75-98.

Binder, H.: Verkabelte Welt. Perspektiven und Probleme der Neuen Medien. (= Medienpraxis, Modelle für die Medienpädagogik 12). Frankfurt o. J.

Binder, H.: Verkabelte Welt. Perspektiven und Probleme der Neuen Medien. Medienpädagogisches Seminar über drei Arbeitseinheiten. Stuttgart 1982.

Boventer, H.: Die Kirche und die Neuen Medien. In: Stimmen der Zeit 199 (1981), S. 775-785.

Boventer, H.: Der elektronische Mensch, Medium und Botschaft - Szenenwechsel für Marshall McLuhan? In: Communicatio Socialis $1 / 1982$, S. 37-47.

Boventer, H.: Ethik und Journalismus: Eine Untersuchung des Hastings Center zur Medienethik im Ausbildungsprogramm an amerikanischen Colleges und Universitäten. In: Communicatio Socialis 4/1982, S. $329-333$.

Boventer, H.: Nachdenken über die Neuen Medien. Die Bedeutung der kommunikationstechnologischen Neuerungen für die pädagogische Situation in Schule und Unterricht. In: Engagement 4/1984, S. 309-319.

Boventer, H.: Angestrengter über Moral nachdenken. Literaturbericht aus USA: Medienanalyse und Medienkritik. In: Communicatio Socialis 1/1986, S. 69-73.

Bröckers, W. (Hrsg.): Die Neuen Medien. Herausforderung an die Kirche. Frankfurt/M. 1984.

Büschges, G.: Soziologische und sozialpolitische Aspekte der Mikroelektronik. In: Karl Goser, Günter Büschges: Mikroelektronik als technische und gesellschaftliche Herausforderung. (= Veröffentlichung der Katholischen Akademie Schwerte, Akademie-Vortrag 13). Schwerte 1984, S. 23-32.

Cover, J.: Theological reflections: Social effects of Television. In: Religious Education 78 (1983), S. 38-49.

Dalferth, Ingolf U.: Kirche in der Mediengesellschaft - Quo vadis? Eine Anfrage. In: Themen der praktischen Theologie - Theologica Practica 2/1985, S. I83-194.

Dörger, H.J.: Gefahren und Chancen der »Neuen Medien«. Verantwortung in Kirche und Gemeinde. In: Religion heute 1/1986, S. 4-10.

Gottschlich, M.: Religion in der Informationsgesellschaft. Versäumnisse, Fehlleistungen und Ansprüche. In: Communicatio Socialis 1/1986, S. 1-16.

Hemmerle, K.: Damit die Zukunft eine Zukunft hat. Maßstäbe und Orientierung. (= Kirche und Gesellschaft 111). Köln 1984

Hilpert, K.: Die Einführung neuer Kommunikationstechniken im Spannungsfeld von Meinungsfreiheit, Sozialität und Gerechtigkeit. In: Religionspädagogische Beiträge 15 (1985), S. 99-125.

Höffner, J. : Dimensionen der Zukunft. Eröffnungsreferat bei der Herbstvollversammlung der Deutschen Bischofskonferenz, Fulda, 20.9.1982. (Der Vorsitzende der DBK 9, hrsg. vom Sekretariat der DBK). Bonn 1982.

Hosse, J.: Kirchen und Kommunikation im ausgehenden 20. Jahrhundert. In: Communicatio Socialis 2/1980, S. $166-170$.

Kerber, W.: Technischer Fortschritt auf Kosten der Ethik? In: Stimmen der Zeit 203 (1985), S. 217 f.

Keul, A.: Science Fiction, Psychologie und Religion. In: Communicatio Socialis 1/1986, S. 33-46.

Lehmann, K.: Das technisch Machbare und das ethisch Verantwortbare. Überlegungen zu einem Grundkonflikt der modernen Zivilisation. Vortrag im Mainzer Dom innerhalb der Reihe »Für eine menschliche Zukunft«am 15.4.1986 (Manuskript).

Lüscher, K.: Menschliche Entwicklung und Entwicklung der Medien, Grundlagen einer Medienökologie. In: Familie und Neue Medien. (= Materialien zur Familienpolitik in NRW, Schriftenreihe Nr. 2). Hrsg. von Landesarbeitsgemeinschaft der Familienverbände in NRW. Münster 1982, S. 7-18. 
Meier, Urs: Der prekäre Übergang zur Informationsgesellschaft. Kommunikation als Thema ethischer Reflexion. In: Reformatio 5/1985, S. 346-352.

Piazolo, P.H.: Die Zukunft der Informationsgesellschaft. Entwicklungen auf dem Gebiet der Informationstechnologie und ihre Bedeutung für das Bildungs- und insbesondere Schulwesen. In: Engagement 4/1984, S. 277-285.

Pöggeler, F.: Zur pädagogischen Problematik der neuen Medien. In: Katholische Bildung 85 (1984), S. 460-469.

Saxer, U.: Bildung und Pädagogik zwischen alten und neuen Medien. In: Media Perspektiven 1983, Heft 1, S. 21-27.

Schmidt-Degenhard, M.: Neue Medien - Alte Fragen? (Rück-)Besinnung auf Elemente kirchlicher Medienverantwortung. In: Caritas 1/1983, S. 1-12.

Schreiber, H.-P.: Wissenschaftsentwicklung als Bedrohung der Menschenwürde. In: Reformatio 3/1985, S. 206-208.

Splett, J.: »Macht euch die Erde untertan?« Zur ethisch-religiösen Begrenzung technischen Zugriffs. In: A. J. Buch/J. Splett (Hrsg.): Wissenschaft - Technik - Humanität. Beiträge zu einer konkreten Ethik. Frankfurt/M. 1982 , S. 175-202.

Wagner, H.: „Die neuen Medien« - Chancen und Gefahren für den Christen. Vortrag zur Herbstvollversammlung des Diözesanrates Eichstätt auf Schloß Hirschberg am 22.9.1984 (Manuskript).

Weizsäcker, C.F. v.: Glaube und wissenschaftlich-technische Welt. (= Aktuelle Akademie, Katholikenausschuß der Stadt Köln). Köln 1980.

Wittkämper, G.W.: Es gibt für die modernen Informations- und Kommunikationstechniken keine Alternativen - auch für die Kirche nicht. (Vortrag im Rahmen des Seminars "Erkennbare Tendenzen der Kommunikation, Alternativen und Konsequenzen« der Kirchlichen Gemeinschaftsstelle für den elektronische Datenverarbeitung e.V., Frankfurt/M., 3.11.1982 in Alzenau-Wasserlos). Maschinenschrift.

2 Vgl. die nachfolgend genannte (ausgewählte) Literatur:

Becker, W.: Familien in der Medienzukunft. In: Caritas 1/1983, S. 12-17.

Betz, G.: Medienpädagogik in der katholischen Erwachsenenbildung. In: Communicatio Socialis 1/1981, S. 50-60.

Betz, G.: Medienerziehung in der Erwachsenenbildung. In: Communicatio Socialis 2/1981, S. 120-130.

Betz, G. : Engagement mit diffuser Programmatik. Die katholische Erwachsenenbildung krankt an ihrer »Theorie火. In: Orientierung Nr. 20 v. 31.10.1984, S. 214-223.

Biser, E.: Der Glaube im Medienzeitalter. Angst und Einsamkeit lassen den Menschen zur Droge Fernsehen greifen. In: academia 4/1984, S. 134-139.

Deutsche Evangelische Arbeitsgemeinschaft für Erwachsenenbildung: Stellungnahme zu den neuen Informations- und Kommunikationstechnologien (*Neue Medien«) vom 14.3.1982.

Diers, A.: Die katholische Erwachsenenbildung. Anmerkungen zu einer freiheitlichen Bildungsposition. In: Erwachsenenbildung 1/1981, S. 25-30.

Emeis, D.: Theologische Überlegungen zu einem Selbstverständnis katholischer Erwachsenenbildung. In: Erwachsenenbildung 17/1971, S. 207-222.

Emeis, D.: Aufgaben der kirchlichen Familienbildungsstätten aus pastoraler Sicht. In: Auftrag und Praxis kath. Familienbildungsstätten und Perspektiven ihrer Arbeit. Düsseldorf 1980, S. 3-27.

Erwachsenenbildung der Kirche, Die: Gemeinsames Papier der Arbeitsstelle der DEAE und der KBE zur UNESCO-Konferenz über Erwachsenenbildung 1985 in Paris.

Kerstiens, L.: Ethische Aspekte der Erwachsenenbildung. In: Erwachsenenbildung 3/1983, S. 160-164.

Leuenberger, Th.: Generation zwischen Krise und Neuorientierung. Die achtziger Jahre - wozu fordern sie heraus? In: Reformatio 7 + 8/1983, S. 313-321. 
Poelchen, H.W.: Sozial soll es sein. Computer, Schule, Weiterbildung. In: Weiterbildung und Medien 3/1986, S. 44-46.

Prokop, E.: Lernen unter Erwachsenen. Didaktik der Erwachsenenbildung bei freien Trägern. München 1983.

Schmid-Degenhard, M.: Neue Medien - Alte Fragen? (Rück-)Besinnung auf Elemente kirchlicher Medienverantwortung. In: Caritas 1/1983, S. 1-12.

Schuchart, A.: Erziehung und Bildung als Verantwortungsbereich des Christen. Aspekte zur theologischen Begründung der kirchlichen Erwachsenenbildung nach dem II. Vatikanischen Konzil. In: Erwachsenenbildung 4/1985, S. 189-192.

Süßmuth, R.: Familienbildung vor neuen Aufgaben - Eigenanspruch und Fremderwartung. In: Auftrag und Praxis kath. Familienbildungsstätten und Perspektiven ihrer Arbeit. Düsseldorf 1980, S. 28-68.

Tuggener, H.: Was hat die Kirche in der Erwachsenenbildung zu suchen? In: Reformatio 7-8/1983, S. 306-313. Vogel, E.: Zur Erwachsenenbildung in kirchlicher Verantwortung. Soziologische Aspekte und gesellschaftliche Einflußfaktoren. In: Erwachsenenbildung 4/1984, S. 237-239.

Zahrnt, H.: Christsein zwischen Hoffnung und Resignation. Die achtziger Jahre - wie antworten wir? In: Reformatio 7-8/1983, S. 321-327.

Zangerle, I.: Christliche Erwachsenenbildung zwischen Frömmigkeit und Mündigkeit. In: Erwachsenenbildung 4/1984, S. 199-202.

Zeitschrift für Weiterbildung in Rheinland-Pfalz: Schwerpunkt: Neue Medien. Heft 3, 1983.

3 Betz, G.: Engagement ..., S. 219.

4 Vogel, E.: Zur Erwachsenenbildung ..., S. 239. 


\section{SUMMARY}

Modern technologies have entailed new conditions for human life. Today many things are practicable and possible, what is not likewise desirable. Responsibilities that have to be worked out considering the Christian ethics' point of view result from this conflict. This is a special duty for the institutions of Catholic adult education. They have the task to accompany people out of Christian values in the orientation crisis caused by modern technology.

\section{RÉSUMÉ}

Les progrès technolgiques ont entrainé de nouvelles conditions de vie pour l'homme. De nos jours, beaucoup de choses sont faisables et possibles qui ne sont pas toujours si souhaitables. Cette contradiction a pour consequence des responsabilités qui doivent être considérées au regard de l'éthique chrétienne.

Cela inflige une charge particulière à l'education catholique des adultes. Celle-ci a le devoir d'aider les hommes à sortir de la crise d'orientation provoquée par la technologie moderne et de les ramener sur la voie des valeurs morales chrétiennes.

\section{RESUMEN}

Las tecnologías modernas trajeron consigo nuevas condiciones a la vida humana. Hoy, mucho es posible y realizable, aunque no igualmente deseable. De estas discrepancias, resultan responsabilidades que tienen que ser tratadas desde un ángulo visual de la tica cristiana. Esto obliga especialmente a las organizaciones católicas para laeducación de adultos. Tienen la tarea de sacar los valores cristianos del hombre, fuera de la crisis de orientación ocasionada por la tecnología moderna. 